\title{
Tratamento dos Desarranjos da Articulação Radio-Ulnal Dis- tal pela Técnica de Sauvé-kapandji. Resultados Preliminares
}

\author{
Treatment of the Distal Radioulnar Articulation Disorders by the Sauvé-kapandji Technique
}

Nilton Mazzer ${ }^{1}$; Cláudio Henrique Barbierl $I^{2}$; Marcia Maradel Pereira Tuma Martins ${ }^{3}$; Andrel Garcia de Souza 3

\section{RESUMO}

Foi realizado um estudo retrospectivo de 10 casos de desarranjo da articulação radio-ulnal distal tratados pela técnica de Sauvé-Kapandji. Os desarranjos eram resultantes de trauma (7), degeneração (2) e doença congênita (1) e as principais queixas pré-operatórias eram a dor, limitação da prono-supinação e deformidade. A idade média dos pacientes na época da operação era de 37,8 anos. Os pacientes foram submetidos a avaliação clínico-funcional e radiografica com um seguimento pós-operatório médio de 28,3 meses, particular atenção tendo sido dada a uma incidência radiográfica anteroposterior sob esforço de preensão manual. Todos os pacientes obtiveram melhora de suas queixas e o resultado final foi considerado satisfatório (excelente e bom) em 8, e insatisfatório (regular) 2. A artrodese da articulação radio-ulnal consolidou em 9 casos, independentemente do tipo de fixação empregado. O exame radiográfico sob esforço mostrou que todos os pacientes apresentavam deslocamento radial da ulna, mas isso aparentemente não interferiu com a função. Concluiuse que a técnica de Sauvé-Kapandji é eficiente para o tratamento dos desarranjos da articulação radio-ulnal distal, pois ela não implica em perda funcional importante.

\section{SUMMARY}

A retrospective study of 10 cases of derrangement of the distal radioulnar joint treated with the technique of SauvéKapandji was carried out. Derrangements resulted from trauma (7), degeneration (2) and congenital disease (1) and the main preoperative complaints were pain, limitation of pronation and supination and deformity. The patients' average age by the time of the operation was 37.8 years (range: 19 - 68 years). All were submitted to clinical, functional and radiographic evaluation at 28.3 months after the operation on average, particular attention being paid to an anteroposterior $X$-ray view with the hand in a moderate hand grip effort. All patients improved from their complaints and the final results were considered satisfactory (excellent or good) in 8, and unsatisfactory (regular) in 2. The distal radioulnar arthrodesis healed uneventfully in 9 cases, independently from the type of fixation used. The X-ray view under strain showed that all patients presented dislocation of the ulna towards the radius, but this apparently did not interfere with function. It was concluded that Sauvé-Kapandji's technique is efficient to treat derrangements of the distal radioulnar joint, since it does not imply significant functional loss.

\footnotetext{
1 Professor Associado

2 Professor Titular

3 Médico residente R4
}

Serviço de Cirurgia da Mão e do Membro Superior e Microcirurgia. Hospital das Clínicas da Faculdade de Medicina de Ribeirão Preto - USP CEP 14048-900 Ribeirõ Preto SP BRASIL 


\section{INTRODUÇÃO}

Os desarranjos da articulação radioulnal distal, seja qual for sua etiologia, comprometem significativamente a função do punho e da mão ${ }^{1}$, uma vez que causam freqüentemente dor, limitação importante da prono-supinação e diminuição da força de preensão ${ }^{1,2}$

As etiologias predominantes dos desarranjos são as lesões traumáticas, degenerativas e congênitas. No primeiro grupo, as mais freqüentes são as seqüelas das fraturas da extremidade distal do rádio e as menos freqüentes, as da fratura de Galeazzi e de outras fraturas do antebraço ${ }^{3,4}$, no segundo grupo, a artrite reumatóide ${ }^{1,2,5}$, e no terceiro, menos freqüente que os dois primeiros, a doença de Madelung e outras ${ }^{1,6}$.

O tratamento desses desarranjos, usualmente crônicos, continua sendo um desafio para os cirurgiões, em virtude da complexidade anatômica e funcional da articulação radio-ulnar distal$^{2}$. Várias técnicas foram propostas para restabelecer a movimentação perdida e aliviar a dor ${ }^{7}$, como por exemplo, as operações de Darrach ${ }^{1}$ (ressecção da ulna distal), de Bowers ${ }^{8}$ (hemirressecção-interposição) e de Baldwin ${ }^{9}$ (ressecção do colo ulnar). A cirurgia de Darrach é melhor indicada apenas para os pacientes idosos, com expectativa de vida não muito longa, desde que o comprometimento da articulação radioulnar distal seja intenso. Já a de Bowers é reservada para pacientes adultos ou idosos que não tenham, porém, lesão importante da fibrocartilagem triangular? ${ }^{7}$. A de Baldwin é, provavelmente, a menos freqüentemente indicada.

Sauvé e Kapandji, em 1936 2,3,10, descreveram a sua técnica, que consiste da fusão da articulação radio-ulnar distal, por cruentação das superfícies articulares e fixação com fios de Kirschner ou parafuso, adicionando a ressecção de um segmento de 10 a 20 $\mathrm{mm}$ da metáfise distal da ulna para que ai se desenvolva uma pseudo-artrose ${ }^{2,7}$

A então chamada operação de Sauvé-Kapandji é indicada para tratar os desarranjos da articulação radio-ulnar distal das mais diversas etiologias, mas que resultem em incongruência, instabilidade e dor, refratárias ao tratamento não operatório ${ }^{2,8,12}$. Ela é, também, um procedimento de salvação para tentativas prévias de tratamento cirúrgico mal sucedido, como a hemirressecçãointerposição de Bowers ${ }^{9}$. As principais contra-indicações da operação são a artrite séptica ativa, o comprimento insuficiente da ulna, como, por exemplo depois de uma operação de Darrach ou de ressecção tumoral, e a ausência da cabeça do radio².

A operação de Sauvé-Kapandji ganhou muitos adeptos nos últimos anos, face aos bons resultados obtidos, principalmente pelo alívio da dor e pelo ganho da prono-supinação ${ }^{8,11}$. Inúmeras vantagens têm sido relatadas, sobretudo quando comparadas à cirurgia de Darrach. Assim, a manutenção da cabeça da ulna propicia a manutenção dos contornos estéticos do punho e impede a luxação do tendão flexor ulnar do carpo, além do que a superfície articular distal da ulna, que é deixada intocada, conserva a transmissão fisiológica de forças entre a mão e o antebraço ${ }^{2,3}$.

\section{INTRODUCTION}

Derrangements of the distal radioulnar joint of any etiology significantly impair the wrist and the hand function', since they frequently cause pain, important limitation of pronation-supination and reduction of grip strenght $t^{1,2}$.

The dearrangements predominant etiologies are the traumatic, degenerative and congenital lesions. In the first group, the most frequent are fracture sequelae in the distal end of the radius and, the less frequent, Galeazzi fracture and other fractures of the forearm ${ }^{3,4}$; in the second group, rheumatoid arthritis ${ }^{1,2,5}$; and in the third, less frequent than the first two groups, Madelung disease and others ${ }^{1,6}$

The usually chronic treatment of these derrangements, continues to challenge the surgeons, in view of the anatomical and functional complexity of the distal radioulnar joint ${ }^{2}$. Several techniques were proposed to re-establish the lost movements and alleviate pain, as for instance, Darrach's ${ }^{1}$ (ressection of the distal ulna), Bowers'8 (hemiressection-interposition) and Baldwin's ${ }^{9}$ (ressection of the ulnar neck) operations. Darrach's surgery is better indicated for elderly patients, with a not very long life expectancy, since the distal radioulnar articulation impairment is intense. Bowers' intervention is reserved for adult or elderly patients not importantly lesioned in the triangular fibrocartilage ${ }^{7}$. Baldwin's is, probably, the less frequently indicated.

Sauvé and Kapandji, in 1936,3,10 described their technique consisting of distal radioulnar articulation fusion by cruentation of the articular surfaces and fixation with Kirschner wires or screws, adding the ressection of a 10 to $20 \mathrm{~mm}$ segment of the ulna distal metaphysis in order that a pseudoarthrosis develops at that point.

The Sauvé-Kapandji operation is indicated to treat dearrangements of the distal radioulnar articulation from several and different etiologies, but resulting in incongruity, instability and pain, refractary to non-surgical treatment ${ }^{2,8,12}$. It is, also, a salvation procedure for previous tentatives of unsuccessful surgical treatment, as the Bowers' hemiressection-interposition ${ }^{9}$. The main contraindications for the operation are active septic arthritis, the ulna insufficient lenght as, for instance, after the Darrach operation or tumoral ressection, and absence of the radius head?

The Sauvé Kapandji operation won several adepts during the last years, face the good results which were obtained, mainly due to pain alleviation and pronation-supination improvementt,11. Several advantagens have been reported mainly when comparing it to Darrach's surgery. Thus, maintenance of the ulna head propitiates maintenance of the esthetic appearance of the wrist and hinders luxation of the carpus ulnar flexor tendon, and the ulna distal articular surface, untouched, conserves the physiological transmission of forces between the hand and the forearm ${ }^{2,3}$.

The main disadvantage, also the most commonly reported complication, is the instability of the ulna proximal segment ${ }^{2,3}$. Nevertheless, it can be corrected during the same operation or, in a later occasion, by fixation of a distal band of the carpus ulnar flexor or extensor tendons, maintained in its original insertion into the ulna 
Por outro lado, a principal desvantagem, que se constitui também na complicação mais comumente relatada, é a instabilidade do segmento proximal da ulna ${ }^{2,3}$. Esta, todavia, pode ser corrigida, no mesmo ato operatório ou tardiamente, pela fixação de uma tira distal do tendão flexor ou extensor ulnar do carpo, que é mantida na sua inserção original, ao coto proximal da ulna, através do seu canal medular. Mais raramente, pode ocorrer ruptura do tendão extensor comum dos dedos ${ }^{13}$.

O objetivo deste trabalho foi estudar retrospectivamente os pacientes operados pela técnica de Sauvé-Kapandji, todos portadores de seqüelas oriundas de diversas etiologias, comparando nossos resultados com os da literatura.

\section{MATERIAL E MÉTODOS}

No período de fevereiro de 1995 a abril de 1999 foram operados dez punhos em dez pacientes portadores de desarranjos da articulação radio-ulnar distal, pela técnica de Sauvé-Kapandji. Seis pacientes eram mulheres e quatro, homens, com idade média de 37,8 anos na ocasião da cirurgia (variação: 19 a 68 anos). O punho direito foi o acometido em sete casos e o intervalo médio entre o início dos sintomas e a intervenção cirúrgica foi de 23,7 meses (variação: 3 a 96 meses). (Tabela 1).

As patologias primárias que ocasionaram o desarranjo da articulação foram a seqüela de trauma, em sete pacientes, lesão degenerativa, em dois, e a paralisia obstétrica, em um. Dos sete casos de seqüela de trauma, cinco (números de ordem 1, 2, 4, 9 e 10) foram secundários a fraturas da extremidade distal do rádio, um (número de ordem 3), a fratura de Galeazzi, e um (número de ordem 5), a fratura diafisária dos ossos do antebraço.

Dos dois punhos com lesão degenerativa (número de ordem 7 e 8), um resultou de artrite reumatóide e o outro de impacto ulnocarpal, após artrodese total do punho. Apenas um paciente (número de ordem 6) teve a operação indicada por seqüela de patologia congênita (paralisia obstétrica), na qual o antebraço encontrava-se fixo em supinação e o punho em desvio radial e extensão. tendon common to the fingers can seldom occur ${ }^{13}$.

The aim of this study was to retrospectively study patients operated on by the Sauvé-Kapandji technique, all carriers of sequelae from several etiologies and compare our results with the literature.

\section{MATERIAL AND METHODS}

From February, 1995, to April, 1999, ten wrists in ten patients carriers of derrangements of the distal radioulnar articulation were operated on by the Sauvé-Kapandji technique. Six female and four male patients with average age 37.8 years by the time of operation (range: 19 to 68 years). The right wrist was impaired in seven cases and the average time between the beginning of the symptoms and the surgical intervention was 23.7 months (range: 3 to 96 months) (Table 1).

The primary pathologies that caused the articulation dearrangement were trauma sequelae in seven patients, degenerative lesion in two, and obstetric paralysis in one. From the seven cases of trauma sequelae, five (numbers of order 1, 2, 4, 9, 10) were secondary to fractures in the distal end of the radius, one (number of order 3) to a Galeazzi fracture and one (number of order 5) to diaphyseal fracture of the forearm bones.

Among the two wrists with degenerative lesion (numbers of order 7 and 8), one resulted from rheumatoid arthritis and the other from ulnocarpal impact, after wrist total arthrodesis. Only one patient (number of order 6) was referred to surgery due to a congenital pathology sequela (obstetric paralysis), with the forearm fixed in supination and the wrist in radial deviation and extension.

The main complaints justifying the indication for Sauvé-Kapandji surgery were limited pronation-supination, pain during usual activities and wrist deformities.

\section{Surgical procedure}

The patients were operated on under regional (8) or general (2) anesthesia. The approach to the distal radioulnar articulation was made through dorsal longitudinal incision on the ulna or triradiate. Initially, the articulation was opened, and then cruentation was made

Tabela 1: Características gerais dos pacientes operados com a técnica de Sauvé-Kapandji

Table 1 : General characteristics of the patients operated on with the Sauvé-Kapandji technique.

\begin{tabular}{|c|c|c|c|c|c|c|c|c|c|}
\hline $\begin{array}{l}\mathbf{N}^{0} \text {. } \\
\text { N. }\end{array}$ & $\begin{array}{l}\text { Sexo } \\
\text { Sex }\end{array}$ & $\begin{array}{l}\text { Idade } \\
\text { Age }\end{array}$ & $\begin{array}{l}\text { Lado } \\
\text { Size }\end{array}$ & $\begin{array}{c}\text { Diagnóstico } \\
\text { Diagnosis }\end{array}$ & $\begin{array}{l}\text { Queixas } \\
\text { Complaints }\end{array}$ & $\begin{array}{c}\text { Dt lesão/S.K. } \\
\text { (meses) } \\
\text { SKLesion. } \\
\text { (Months) } \\
\end{array}$ & $\begin{array}{l}\text { Fixação } \\
\text { Fixation }\end{array}$ & $\begin{array}{l}\text { Cirurgia associada } \\
\text { Associate surgery }\end{array}$ & $\begin{array}{l}\text { Seguim. } \\
\text { (meses) } \\
\text { Follow-up } \\
\text { (Months) }\end{array}$ \\
\hline 1 & M & 26 & $E$ & F. Rád. Dist. & Dim. P.S. & 33 & 1 parafuso & Estabil. Ulna c/ EUC & 8 \\
\hline 2 & $\mathrm{~F}$ & 68 & $E$ & F. Rád. Dist. & Dor+Dim. P.S. & 10 & 1 parafuso & - & 57 \\
\hline 3 & M & 22 & $\mathrm{D}$ & F. Galeazzi & Def +Dim. P.S. & 6 & 1 parafuso & Estabil. Ulna c/ EUC & 9 \\
\hline 4 & $\mathrm{~F}$ & 22 & D & F. Rád. Dist.+ L. Tend. Nerv. & Def.+Dim. P.S. & 11 & 2 fios $\mathrm{K}$. & Osteot. Rádio+Tenólise & 21 \\
\hline 5 & $\mathrm{~F}$ & 45 & $\mathrm{D}$ & F. Diaf. Rád. Ulna & Dor+Dim. P.S. & 9 & 1 parafuso +1 fio $K$ & - & 45 \\
\hline 6 & $\mathrm{~F}$ & 19 & D & Paralisia. Obstétrica & Dor+Def.+Dim. P.S. & $?$ & 1 parafuso+1 fio $K$ & - & 18 \\
\hline 7 & $\mathrm{~F}$ & 49 & D & Impacto Ulno-carpal & Dor+Dim. P.S. & 96 & 1 parafuso +2 fios $K$. & - & 19 \\
\hline 8 & $\mathrm{~F}$ & 68 & D & Artrite Reumatóide & Dor+Def.+Dim. P.S. & $?$ & 1 parafuso & Artrod. Punho+enxerto RUD & 28 \\
\hline 9 & $M$ & 39 & $E$ & F. Rád. Dist. & Dor+Def.+Dim. P.S. & 3 & 1 paraf. +1 fio $K$ & Artrodese Punho & 20 \\
\hline 10 & M & 20 & D & F. Rád. Dist.E1 & $\mathrm{Dim}+\mathrm{F} 12$ & 69 & 2 fios $\mathrm{K}$. & - & 58 \\
\hline
\end{tabular}

Legenda: F:fratura; Rád:rádio;Uln:ulna; Dist: distal; L:lesão; Tend:tendinosa; Nerv:nervosa; Dim:diminuição; P.S.:pronosupinação

Legend: F: fracture; Rád: radius; UIn: ulna; Distal: distal; L: lesion; Tend: tendinous; Ner: nervous; P.S.: pronation-supination 
As principais queixas que fundamentaram a indicação da cirurgia de Sauvé-Kapandji foram a limitação da prono-supinação, a dor às atividades habituais e a deformidade do punho.

\section{Procedimento cirúrgico}

Os pacientes foram operados sob anestesia regional (8) ou geral (2). A abordagem à articulação radio-ulnar distal foi feita através de incisão longitudinal dorsal sobre a ulna ou trirradiada. Inicialmente, era realizada a abertura da articulação, pela secção do ligamento radio-ulnar dorsal, seguindo-se a cruentação das superfícies articulares, até que se visualizasse o osso esponjoso, logo abaixo do osso subcondral. Em seguida, era feita a osteotomia de ressecção de um segmento de osso metafisário de, cujo comprimento médio foi de 11,7 mm (variação: 10 a $17 \mathrm{~mm}$ ), na região do colo da ulna. Opcionalmente, a osteotomia era realizada antes da abertura, cruentação e fixação da articulação. Uma vez feita, ele permite o completo manuseio da cabeça da ulna, facilitanto o seu posicionamento que, idealmente, deve deixá-la na mesma altura da superfície articular radio-cárpica, prevenindo o choque com o osso piramida. Uma vez posicionada, a cabeça da ulna era fixada ao rádio, o que foi feito pela compressão interfragmentária com um parafuso de 3,5 mm de diâmetro, em quatro pacientes, com um parafuso associado a um fio de Kirschner, em outros quatro, e com dois fios de Kirschner paralelos, em dois. Procedimento associados foram realizados em cinco punhos, constando da estabilização do segmento proximal da ulna com uma tira do tendão extensor ulnar do carpo (2), artrodese total do punho (2) e osteotomia corretiva do rádio e tenólise dos tendões flexores (1). Enxerto ósseo foi empregado em apenas um caso, para melhorar o contato entre as superfícies articulares a serem artrodesadas, tendo sido usado como fonte o segmento ressecado da ulna.

No pós-operatório imediato, os pacientes foram imobilizados com uma tala gessada antebraquio-palmar por um período médio de 5,4 semanas (variação: 2 a 6 semanas), sendo depois encaminhados à fisioterapia.

\section{Reavaliação dos pacientes}

O período médio de seguimento na presente reavaliação foi de 28,3 meses (variação: 9 a 58 meses), tendo sido utilizado um protocolo baseado na avaliação clínica, incluindo a medida goniométrica da amplitude de movimentos do punho e antebraço, particularmente a prono-supinação, e a aferição da força de preensão manual, com um dinamômetro. A avaliação radiográfica realizada compreendeu as incidências convencionais (posteroanterior e lateral do punho) e de uma incidência postero-lateral com o paciente fazendo uma preensão manual, segurando um pêso de $2 \mathrm{Kg}$. As duas incidências postero-laterais foram realizadas com o membro superior totalmente em contato com a superfície da mesa radiográfica e o cotovelo fletido a $90^{\circ}$.

Além da avaliação objetiva clínico-radiográfica, foi realizada uma avaliação subjetiva, por parte tanto do paciente, como do médico, constando basicamente da satisfação com o resultado funcional e de eventuais queixas, desde que fundamentadas. of the articular surfaces until the spongy bone was visible, just under the subcontral bone. Afterwards, ressection osteotomy was made of a metaphyseal bone segment with $11.7 \mathrm{~mm}$ (range: 10 to $17 \mathrm{~mm}$ ) average lenght, in the ulna neck region. Optionally, osteotomy was carried out before opening, cruentation and fixation of the joint. When it is carried out complete handling of the ulna head is possible, easing its positioning that, ideally, should be at the same height of the radiocarpal articular surface, preventing shock with the pyramidal bone. After positioned, the ulna head was fixed to the radius in four patients by interfragmentary compression with a $3.5 \mathrm{~mm}$ diameter screw, in other four with a screw associated to a Kirschner wire, and in two with two parallel Kirschner wires. Associate procedures were carried out in five wrists: stabilization of the ulna proximal segment with a band of the carpus ulnar extensor tendon (2), wrist total arthrodesis (2), and corrective osteotomy of the radius and tenolysis of the flexor tendons (1). Bone grafting was used in only one case to improve contact among the articular surfaces to be arthrodesed, having the ulna resected segment as source.

In the immediate post-operatory period, the patients were immobilized with an antebrachial-palmar plaster cast during a mean period of 5.4 weeks (range: 2 to 6 weeks) and were referred to physiotherapy.

\section{Re-evaluation of the patients}

The mean follow-up period in this re-evaluation was 28.3 months (range: 9 to 58 months) and a protocol based in the clinical evaluation was used, including goniometric determinations of movement amplitude of the wrist and forearm, particularly pronation-supination, and the measurement of the manual grip force with a dynamometer. The radiographic evaluation included conventional incidences (posterior-anterior and lateral of the wrist) and a posterior-lateral incidence with the patient exerting manual grip by holding a $2 \mathrm{~kg}$ weight. The two posterior-lateral incidences were carried out with the upper limb totally contacting the X-rays table and the elbow bending $90^{\circ}$.

Besides the clinical-radiographic objective evaluation a subjective evaluation was made, both by the patients and the doctor, aiming basically satisfaction with the functional result and occasional complaints, provided they were founded.

The X-rays were evaluated according to the technique described by Nakamura et al. ${ }^{14}$, modified according to the purposes of this study. Ulnar flaw, resulting from segmental resection and correspondent to the extension of the created pseudoarthrosis, and the radioulnar distance, between the middle point of the ulna diaphysis, immediately proximal to the stump irregularity, and the middle point of the radius diaphysis, at the same height (Figure 1). That distance was measured in the conventional posterior-anterior incidences and with load, and the difference between the two results was called ulnar displacement in the radius direction, that is, it is the ulna instability quantification in the radioulnar plan.

The results were classified as satisfactory (excellent and good) or non-satisfactory (regular and bad), and this was a subjective determination, since the sample was small and heterogeneous, as a function of the different etiologies. 
Os exames radiográficos obtidos foram avaliados segundo a técnida descrita por Nakamura e colaboradores ${ }^{14}$, modificada para os propósitos deste trabalho. Assim, eram medidas a falha ulnar, resultante da ressecção segmentar e correspondente à extensão da pseudoartrose criada, e a distância radio-ulnar, entre o ponto médio da diáfise da ulna, imediatamente proximal à irregularidade do coto, e o ponto médio da diáfise do rádio, na mesma altura (Figura 1). Essa distância foi medida nas incidências em posteroanterior convencional e com carga, e a diferença entre os dois valores obtidos foi denominado de deslocamento ulnar na direção do rádio, ou seja, é a quantificação da instabilidade da ulna no plano radio-ulnar.

Os resultados foram classificados como satisfatórios (excelente e bom) ou insatisfatórios (regular e mau), tendo sido esta uma determinação subjetiva, uma vez que a amostra de pacientes era pequena e heterogênea, em função das etiologia diversas apresentadas.

\section{RESULTADOS}

A avaliação final dos pacientes baseou-se fundamentalmente na sintomatologia remanescente, no retorno ou não às atividades anteriores e na opinião do paciente, levando-se também em consideração a gravidade da patologia de base.

Todos os pacientes obtiveram algum grau de melhora após a intervenção. Na avaliação global, oito pacientes tiveram resultados classificados de satisfatórios, sendo cinco excelentes e três bons, e dois pacientes tiveram resultados insatisfatórios, por terem sido considerados apenas regulares. Nenhum resultado foi considerado francamente mau.

\section{Resultados clínicos}

Dor: cinco pacientes não relataram mais nenhuma dor, enquanto que três referiram dor apenas aos esforços pesados, e apenas um aos esforços.

Mobilidade: a prono-supinação medida foi, em média, de $148^{\circ}$, ou $90 \%$ do normal, variando de $112^{\circ}$ a $175^{\circ}$ (67\% a $100 \%$ do normal). A flexo-extensão do punho variou de $66^{\circ}$ a $144^{\circ}$ (40\% a $90 \%$ do normal), com valor médio de $104^{\circ}$, ou $40 \%$ do normal, não tendo sido considerados aqui os pacientes com artrodese total punho (números de ordem 7,8 e 9). (Tabela 2).

Força de preensão: foi em média de 16 Kgf, representando 68\% do lado normal contralateral. A variação foi de 4 a $30 \mathrm{Kgf}$, correspondentes, respectivamente, a 20\% e $115 \%$ do normal. Em dois pacientes (números de ordem 7 e 8), a força superou a do lado oposto, uma vez que ambos apresentavam a mesma patologia no punho contralateral (doença de Kienböck e artrite reumatóide, respectivamente), ainda não tratado (Tabela 3).

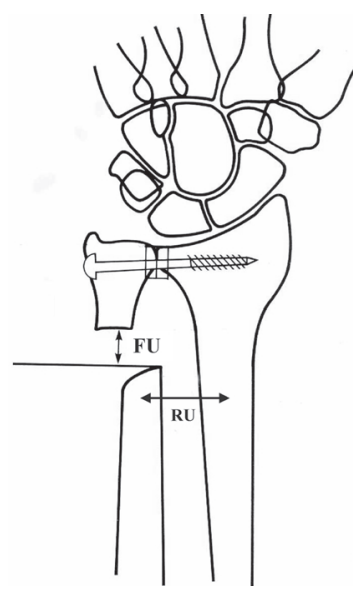

Figura 1 - Esquema das medidas tomadas nas radiografias em póstero-anterior, com e sem carga: FU - falha ulnar;

$\mathrm{RU}$ - distância rádio ulnar (modificado de Nakamura et al. ${ }^{14}$ )

Figure 1 - Diagram of the measures taken in posterior-anterior X-rays with and without load: FU - ulnar flaw; $R U$ - radioulnar distance (modified from Nakamura et al. ${ }^{14}$ ).

\section{RESULTS}

The final evaluation of the patients was fundamentally based in the remaining symptomatology, resuming or not the previous activities and in the patients' opinion, considering also the severity of the pathology.

All the patients showed some grade of improvement after the intervention. In the overall evaluation, eight patients had results considered satisfactory, five excellent and three good, and two patients presented non-satisfactory results, since they were only regular. No manifestedly bad results were obtained.

Tabela 2 - Resultados da avaliação da mobilidade ativa do punho Table 2: Results of the evaluation of the active mobility of the wrist.

\begin{tabular}{|c|c|c|c|c|c|c|}
\hline & \multicolumn{3}{|c|}{$\begin{array}{l}\text { Flexão + Extensão } \\
\text { Flexion + Extension }\end{array}$} & \multicolumn{3}{|c|}{$\begin{array}{c}\text { Pronação + Supinação } \\
\text { Pronation + Supination }\end{array}$} \\
\hline & $\begin{array}{l}\text { Direito } \\
\text { Right }\end{array}$ & $\begin{array}{l}\text { Esquerdo } \\
\text { Left }\end{array}$ & $\begin{array}{l}\% \text { lesado/são } \\
\% \text { lesioned/healthy }\end{array}$ & $\begin{array}{l}\text { Direito } \\
\text { Right }\end{array}$ & $\begin{array}{c}\text { Esquerdo } \\
\text { Left }\end{array}$ & $\begin{array}{l}\text { \% lesado/são } \\
\% \text { lesioned/healthy }\end{array}$ \\
\hline 1 & $130^{\circ}$ & $1110^{\circ *}$ & $84,60 \%$ & $170^{\circ}$ & $160^{\circ *}$ & $94,00 \%$ \\
\hline 2 & $140^{\circ}$ & $115^{\circ} *$ & $82,00 \%$ & $180^{\circ}$ & $175^{\circ} *$ & $97,00 \%$ \\
\hline 3 & $144^{\circ *}$ & $150^{\circ}$ & $96,00 \%$ & $160^{\circ}$ * & $160^{\circ}$ & $100,00 \%$ \\
\hline 4 & $66^{\circ} *$ & $164^{\circ}$ & $40,00 \%$ & $118^{\circ}$ * & $176^{\circ}$ & $67,00 \%$ \\
\hline 5 & $120^{\circ}$ * & $150^{\circ}$ & $80,00 \%$ & $160^{\circ} *$ & $170^{\circ}$ & $94,00 \%$ \\
\hline 6 & P.O. * & $140^{\circ}$ & P.O & P.O. * & $180^{\circ}$ & P.O. \\
\hline 7 & artrodese & artrodese & - & $134^{\circ}$ * & $138^{\circ}$ & $97,00 \%$ \\
\hline 8 & artrodese* & $90^{\circ}$ & - & $160^{\circ}$ * & $165^{\circ}$ & $97,00 \%$ \\
\hline 9 & $130^{\circ}$ & artrodese * & - & $160^{\circ}$ & $160^{\circ} *$ & $100,00 \%$ \\
\hline 10 & $68^{\circ} *$ & $140^{\circ}$ & $48,00 \%$ & $112^{\circ *}$ & $156^{\circ}$ & $71,00 \%$ \\
\hline
\end{tabular}

* lado submetido à cirurgia de Sauvé-Kapandji

* side submitted to Sauvé-Kapandji surgery

P.O.: paralisia obstétrica

P.O. : obstetric paralysis 


\section{Resultados radiográficos}

A artrodese da articulação radio-ulnar distal ocorreu em 9 dos 10 punhos (Figura 2). No único caso em que não ocorreu (Figura 3, número de ordem 4), não foi realizado nenhum procedimento adicional, dada a ausência de dor ou instabilidade da cabeça da ulna.

Em todos os casos, a ressecção segmentar evoluiu para pseudo-artrose e esta falha ulnar mediu de 10 a $17 \mathrm{~mm}$, com média de 11,7 mm (Tabela 4). A incidência em postero-anterior convencional demostrou que a distância radio-ulnar média foi de 18,8 mm (variação: 11 a 26 mm). Com a carga de $2 \mathrm{Kg}$, diminuiu para 12,75 mm, em média (variação: -5,5 a $21 \mathrm{~mm}$ ). Dados esses valores, o deslocamento ulnar em direção do rádio foi observado em todos os pacientes, com valor médio de 6,05 mm (variação: 1 a $16,5 \mathrm{~mm}$ ), traduzindo a instabilidade do coto proximal da ulna no plano radioulnar.

A presença da instabilidade do antebraço não foi motivo de queixa específica dos pacientes e aparentemente não influenciou nos resultados clínicosfinais (Tabela 4).

\section{Complicações}

Três pacientes apresentaram algum tipo de complicação. Num caso, houve infecção superficial, resolvida com antibioticoterapia sistêmica, e instabilidade dorsal assintomática do coto proximal da ulna (número de ordem 1). Em outro, (número de ordem 2), houve consolidação parcial da osteotomia da ulna que, todavia, não necessitou de re-intervenção. No terceiro, (número de ordem 7), ocorreu instabilidade dolorosa do coto proximal da ulna, para a qual foi indicado um procedimento de estabilização com uma tira do tendão extensor ulnar do carpo, ainda não aceita pela paciente.

Oito paciente retornaram às atividades habituais ou profissionais anteriores. Dos dois que não voltaram, um (número de ordem 10) alegou que a lesão, por ser no membro dominante, impedia-o de trabalhar. O outro (número de ordem 7) era portador de artrodese total de ambos os punhos e já não exercia nenhuma atividade habitual ou profissional, limitando-se às atividades pessoais de higiene e alimentação.

\section{DISCUSSÃO}

A técnica de Sauvé e Kapandji para o tratamento dos desarranjos da articulação radio-ulnar distal não é de aceitação universal. Entretanto, séries maiores do que a analisada neste trabalho têm mostrado que ele é eficiente para resolver aqueles problemas, com uma incidência relativamente baixa de complicações ${ }^{1,3,4}$, e os resultados aqui obtidos ratificam esse fato, que tem sido aceito e divulgado na literatura.

O número de resultados satisfatórios aqui obtidos (8/10) se equivalem aos cerca de $80 \%$ relatados por Condamine e Aubriot ${ }^{1}$, que avaliaram 69 pacientes, portadores de patologias de diversas etiologias e pertencentes a diferentes faixas etárias ( $2^{a}$ à $8^{a}$ décadas), tratados com a técnica de Sauvé-Kapandji.
Tabela 3: Força de preensão (Kgf)

Table 3: Grip strenght (Kgf)

\begin{tabular}{cccc}
\hline & $\begin{array}{c}\text { Direito } \\
\text { Right }\end{array}$ & $\begin{array}{c}\text { Esquerdo } \\
\text { Left }\end{array}$ & $\begin{array}{c}\text { \% Lado } \\
\text { \% side Lesiolsand/healtity }\end{array}$ \\
\hline \hline $\mathbf{1}$ & 42 & $12,6^{*}$ & $30 \%$ \\
$\mathbf{2}$ & 27,3 & $25,3^{*}$ & $93 \%$ \\
$\mathbf{3}$ & $27^{*}$ & 33,3 & $81 \%$ \\
$\mathbf{4}$ & $8,3^{*}$ & 21,3 & $39 \%$ \\
$\mathbf{5}$ & $12,6^{*}$ & 17 & $74 \%$ \\
$\mathbf{6}$ & $4^{*}$ & 20 & $20 \%$ \\
$\mathbf{7}$ & $18,5^{*}$ & 16 & $115 \%$ \\
$\mathbf{8}$ & $8^{*}$ & 7,3 & $109 \%$ \\
$\mathbf{9}$ & 40 & $30 *$ & $75 \%$ \\
$\mathbf{1 0}$ & $12,3 *$ & 24,6 & $50 \%$ \\
\hline
\end{tabular}

* lado submetido à cirurgia de Sauvé-Kapandji

* side submitted to Sauvé-Kapandki surgery

\section{Clinical Results}

Pain: five patients did not report any pain, while three informed pain only during intense effort, and one pain with effort.

Mobility: pronation-supination was on average $148^{\circ}$, or $90 \%$ normal, from $112^{\circ}$ to $175^{\circ}$ (67\% to $100 \%$ normal). Flexure-extension of the wrist varied from $66^{\circ}$ to $144^{\circ}$ (40\% to $90 \%$ of normal), averaging $104^{\circ}$, or $40 \%$ normal, however the patients with total wrist arthrodesis were not considered (numbers of order 7, 8 and 9) (Table 2).

Grip strenght: on average $16 \mathrm{Kgf}$, representing $68 \%$ of the normal contralateral side. Range was 4 to $30 \mathrm{Kgfcorresponding,} \mathrm{respectively,}$ to $20 \%$ and $115 \%$ of normal. In two patients (numbers of order 7 and 8), strenght exceeded the opposite side, since both presented the same pathology in the contralateral wrist (Kienböck disease and rheumatoid arthritis, respectively), not yet treated (Table 3).

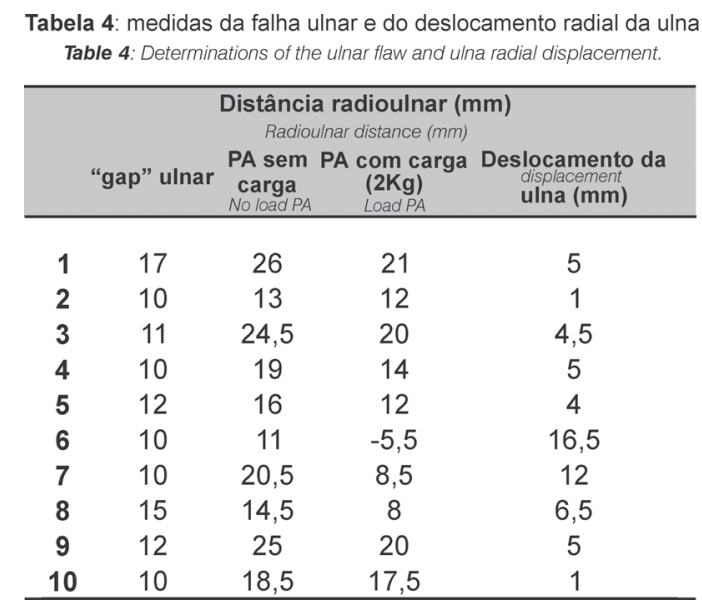


Do mesmo modo que o relatado nas maiores séries ${ }^{1,3,4}$, nos desarranjos da articulação radio-ulnar de origem traumática, o sintoma mais determinante na indicação da operação de SauvéKapandji foi a limitação da prono-supinação, presente em cerca de $90 \%$ dos casos, enquanto que a dor só esteve presente em $43 \%$ dos casos. No que diz respeito a patologias degenerativas, como a da artrite reumatóide, a dor assume maior importância, como é comumente observado ${ }^{1,5}$. Tal fato ocorreu no único paciente dessa patologia operado na presente série, cuja queixa principal era justamente a dor.

A aplicação da técnica de Sauvé-Kapandji para um caso de seqüela de paralisia obstétrica (número de ordem 6) teve como único objetivo o alivio a dor, particularmente em repouso. Tal objetivo foi atingido, e a dor passou a manifestar-se somente aos esforços excessivos. Não houve, ainda, nenhum ganho funcional ou estético, pois o paciente continuou com pouca movimentação e com o antebraço em supinação, ditados pela patologia de base e pela atrofia muscular pré-existente. Essa indicação foi, provavelmente, pioneira, visto que nenhum dos autores consultados referiu tal indicação.

A indicação e a execução adequadas desta técnica conduziram ao o alívio da dor e ao ganho de mobilidade, sobretudo da pronosupinação, possibilitando à maioria dos pacientes o retorno às suas atividades laborativas. É verdade, porém, que em alguns casos a persistência da dor aos esforços pesados, embora bem tolerada, restringiu algumas atividades, fato também observado por outros autores ${ }^{1,5}$.

Os diferentes modos de fixação utilizados para a artrodese da articulação radio-ulnar distal não influenciaram na sua consolidação, uma vez que esta ocorreu na grande maioria dos casos (9/10). No único paciente em que ele não ocorreu, segundo o exame radiográfico, a ausência de sintomas levou à indicação de mera observação periódica sem sugestão de re-intervenção. Quanto ao uso de enxerto ósseo, sugerido por alguns autores ${ }^{2}$, parece ser desnecessário, pois foi empregado uma única vez (número de ordem 8) e, mesmo assim, apenas para melhorar a adaptação da cabeça da ulna ao leito da superfície articular do rádio.

Em sintonia com o que tem sido relatado ${ }^{1,2,3}$, a principal sede de complicações é o segmento proximal da ulna, que permaneceu instável em direção dorsal em dois dos dez pacientes (número de ordem 1 e 7), sendo que num deles era indolor. Naquele em que a instabilidade era dolorosa e anti-estética (número de ordem 7), a provável causa disso foi a falha ulnar muito ampla (17 mm). A exemplo do que ocorreu com a maior parte dos pacientes aqui analisados, existe a recomendação de que o segmento ressecado da ulna situe-se entre 10 e 12 mm de comprimento², além do que a região da ressecção deve permanecer dentro da faixa de origem do músculo pronador quadrado na ulna.

A instabilidade estática e dinâmica da ulna no plano radio-ulnar foi avaliada pela comparação entra as incidências radiográficas convencional e com carga, como proposto por Nakamura et $\mathrm{a}^{14}$. Observou-se então que em alguns casos, mesmo sem fazer carga, a ulna já apresenta uma pequena aproximação com o

\section{X-rays results}

Distal radioulnar articulation arthrodesis was made in 9 among the 10 wrists (Figura 2).

The left out case (Figure 3, number of order 4), had no additional procedures carried out, since pain and instability in the ulna head were absent.

In all the cases, the segment resection evolved to pseudoarthrosis and this ulnar flaw measured 10 to $17 \mathrm{~mm}, 11.7 \mathrm{~mm}$ on average (Table 4). The conventional posterior-anterior incidence evidenced that the average radioulnar distance was $18.8 \mathrm{~mm}$ (range: 11 to 26 $\mathrm{mm}$ ). With the $2 \mathrm{~kg}$ load, it was reduced to $12.75 \mathrm{~mm}$ (range: -5.5 to $21 \mathrm{~mm}$ ). Considering these values, the ulnar displacement in the direction of the radius was observed in all the patients, 6.05 on average (range: 1 to 16.5), evidencing instability of the ulna proximal stump in the radioulnar plan.

The presence of forearm instability was not a specific complaint of the patients and apparently did not influence the final clinical results (Table 4).

\section{Complications}

Three patientes presented complications. In one case, superficial infection, treated with systemic antibioticotherapy, and asymptomatic dorsal instability of the ulna proximal stump (number of order 1). In another (number of order 2), there was partial consolidation of the ulna osteotomy but re-intervention was not necessary. In the third (number of order 7), painful instability occurred in the ulna proximal stump, and a stabilization procedure was indicated with a band of the carpus ulnar extensor tendon, not yet consented by the patient.

Eight patients resumed their previous usual or professional activities. One (number of order 10) alleged that the lesion, in the dominant member, hindered him from working. The other (number of order 7) submitted to total arthrodesis in both wrists had no usual or professional activity, being limited to activities as hygiene and alimentation.

\section{DISCUSSION}

The Sauvé and Kapandji technique for the treatment of the distal radioulnar articulation derrangements is not universally accepted. However, samples greater than the one analyzed in this study, have shown its efficiency to solve these conditions with a relatively low incidence of complications ${ }^{1,3,4}$ and the results of this study confirm that observation, accepted and published in the literature.

The number of satisfactory results obtained in this study (8/10) is equivalent to the approximately $80 \%$ reported by Condamine and Aubriot ${ }^{1}$, evaluating 69 patients with several pathologies of different etiologies and belonging to different age groups (twenties to eighties) treated by the Sauvé-Kapandji technique.

Just as reported with big groups of patients ${ }^{1,3,4}$, in the traumatic radioulnar articulation dearrangements the most determinant symptom to indicate the Sauvé-Kapandji operation was limitation of pronation-supination, present in about $90 \%$ of the mentioned cases, 
rádio. Todavia, ao fazer o esforço para segurar a carga de $2 \mathrm{Kg}$, o franco deslocamento da ulna na direção do rádio ocorreu em todos os casos, caracterizando uma instabilidade entre o rádio e a ulna, a qual não interferiu no resultado final. A relevância dessa investigação estava justamente em determinar a necessidade de se realizar rotineiramente um procedimento de estabilização da ulna, o que parece não ser o caso, considerando a ausência de sintomas, pelo menos no período pós-operatório estudado.

A consolidação parcial da pseudo-artrose ou re-ossificação da osteotomia da ulna é freqüentemente relatada, muitas vezes requerendo reintervenção para corrigí-la ${ }^{1}$. Na presente série, tal fato foi observado em apenas um paciente (número de ordem 2), mas, talvez por envolver apenas $20 \%$ da pseudo-artrose, não chegou a bloquear a prono-supinação e não requereu re-intervenção.

A associação da operação de Sauvé-Kapandji com a artrodese total do punho não é relatada com freqüência. Na presente série, ela foi empregada em três pacientes (números de ordem 7, 8 e 9), tendo produzido resultados satisfatórios em dois, sobretudo por ter permitido quase total equivalência da prono-supinação em relação ao lado oposto. De fato, a artrodese total do punho, realizada por técnicas convencionais, como por exemplo a técnica A.O., não interfere com a articulação radio-ulnar, não corrigindo, portanto, eventuais bloqueios da prono-supinação. Concluiu-se disso que a associação entre aquelas duas técnicas pode ser útil nesses casos e deve ser indicada.

\section{CONCLUSÃO}

Apesar de a presente série ser relativamente pequena e conter casos de desarranjo da articulação radio-ulnar distal de diferentes etiologias, os resultados obtidos, ainda que preliminares, são animadores em relação à aplicabilidade da técnica de SauvéKapandji e da sua eficácia para tratar problemas tão difíceis. Ficou confirmado que essa operação é uma valiosa, opção sobretudo para o tratamento de pacientes jovens portadores de seqüelas de traumatismos, para as quais procedimentos mais radicais, como o de Darrach, seriam mais incapacitantes. Sem estabelecer comparações com outros procedimentos, como por exemplo o de Bowers, a operação de Sauvé-Kapandji é uma arma terapêutica eficaz, cujo emprego deve ser preconizado para casos semelhantes àqueles aqui demonstrados. Entretanto, apesar de relativamente simples e de fácil execução, deve ser realizado com precisão para que os bons resultados se repitam. while pain has only been present in $43 \%$ of the cases. Concerning degenerative pathologies, as rheumatoid arthritis, the evidences show that pain assumes greater importance ${ }^{1,5}$. In this group, this was observed in the only patient with that condition, being pain the main complaint.

The reason for the application of the Sauvé-Kapandji technique in the obstetric paralysis case (number of order 6) was to relieve pain, particularly at rest. That aim was achieved, and pain was observed only during excessive effort. Up to this moment, no functional or esthetic improvement was observed, since the patient continued to present scarce movement with the forearm in supination, consequent to the basic pathology and pre-existent muscular athrophy. That was, probably, a pioneer indication, since none of the revised authors had mentioned that indication.

Adequate indication and execution of this technique led to pain relief and increased mobility, mainly in pronation-supination, possibilitating to most patients to resume their working acitivities. It is true, though, that in some cases pain persistence to heavy effort, though well tolerated, restricts some activities, and this was also mentioned by other authors ${ }^{1,5}$

The different fixation methods used for the distal radioulnar articulation arthrodesis did not influence its consolidation, since this was observed in most of the cases (9/10). In the patient consolidation did not occur as X-rays view has evidenced, the absence of symptoms indicated periodic observation and re-intervention was not considered.

The use of bone grafts, suggested by some authors'2, seems unnecessary since it was used in only one occasion (number of order 8) aiming exclusively to improve adaptation of the ulna head to the radius articular surface bed.

In agreement with the other studies ${ }^{1,2,3}$, the main site of complications is the ulna proximal segment, that remained dorsally unstable in two of the ten patients (numbers of order 1 and 7), one of them painless. In the patient with painful and anti-esthetic instability (number of order 7 ), the probable cause was a very wide ulnar flaw $(17 \mathrm{~mm})$. Similarly to most of the patients of this study, it is recommended that the resected ulna segment is 10 to $12 \mathrm{~mm}$ long $^{2}$, and that the resection region be located inside the band of origin of the ulna squared pronator muscle. Static and dynamic instability of the ulna in the radioulnar plan was evaluated comparing the conventional radiographic incidences and load, as proposed by Nakamura et al. ${ }^{14}$. In some cases, even with no load, the ulna presents small approximation to the radius. However, when effort is made to hold the $2 \mathrm{~kg}$ load, manifested displacement of the ulna in the direction of the radius occurred in all cases, characterizing an instability between the radius and the ulna, which did not interfere in the final result. The relevance of this investigation was to determine the need to routinely carry out a ulna stabilization procedure and this apparently is not the case considering the absence of symptoms at least in the post-surgery studied period.

Partial consolidation of pseudoarthrosis or re-ossification of the ulna osteotomy are frequently reported, often demanding corrective re-intervention'. In this group of patients, this was observed in only one patient (number of order 2) but maybe because only $20 \%$ of the 


\section{REFERÊNCIAS}

1. Condamine J. L., Lebreton L.,Aubriot J. H.: L'intervention de SauvéKapandji. Analyse et résultats de 69 cas. Ann. Chir. Main 11(1): 27-39, 1992.

2. Slater R. R., Szabo R. M.: The Sauvé-Kapandji Procedure. Tech. Hand Upper Limb Surg. 2(3): 148-157, 1998

3. Mikkelsen S. S., Lindblad B. E., Larsen E. R., Sommer J.: SauvéKapandji operation for disorders of the distal radioulnar joint after Colles' fracture. Acta Orthop. Scand. 68: 64-66, 1997.

4. Mestdagh H., Duquennoy A., Letendart J., Sensey J. J., Fontaine C. Résultats à long terme du traitement des fractures-luxations de Galeazzi chez l'adulte. Ann. Chir. Main 2(2): 126-133, 1983

5. Tran Van F., Obry Ch., Fardellone P., Deccoppman M., Vives P. Réhabilitation du poignet dorsal rhumatoïde par l'intervention de SauvéKapandji associée à une synovectomie-rélaxation-stabilisation. Ann. Chir. Main 12(2): 115-123, 1993.

6. Angelini L. C., Leite V. M., Faloppa F.: Surgical treatment of Madelung disease by the Sauvé-Kapandji technique. Ann. Chir. Main 15(4): 257-264, 1996.

7. Minami A., Suzuki K., Suenaga N., Ishikawa J.: The Sauvé-Kapandji procedure for osteoarthritis of the distal radioulnar joint. J. Hand Surg. 20A: 602-608, 1995.

8. Gonzalez Del Pino J., Fernandez D. L.: Salvage procedure for failed Bowers' hemiresection interposition technique in the distal radioulnar joint. J. Hand Surg. 23B: 749-753, 1998

9. Kersley J. B., Scott W.: Restoration of forearm rotation following malunited fractures: Baldwin's operation. J. Hand Surg. 15B: 421-424, 1990

10. Ladd A. L., Huene D. S.: Reconstructive osteotomy for malunion of the distal radius. Clin. Orthop. 237: 158-171, 1996.

11. Usui M., Murakami T., Naito T., Wada T., Takahashi T., Ishii S.: Some problems in wrist reconstruction after tumor resection with vascularized fibular-head graft. J. Reconstr. Microsurg. 12: 81-88, 1996.

12. Blanco R., Blanco F.: The use of a bone peg in the Sauvé-Kapandji operation. J. Hand Surg. 19B: 221-223, 1994.

13. Wada T., Ogino T., Ishii S.: Closed rupture of a finger extensor tendon following the Sauvé-Kapandji procedure: a case report. J. Hand Surg. 22 A: 705-707, 1997

14. Nakamura R., Tsunoda K., Watanabe K., Horii E., Miura T.: The SauvéKapandji procedure for chronic dislocation of the distal radioulnar joint with destruction of the articular surface. J. Hand Surg. 17B: 127-132, 1992 pseudoarthrosis was involved, it did not block pronation-supination and did not require re-intervention.

The association of the Sauvé-Kapandji operation with wrist total arthrodesis is not frequently reported. In this series of patients, it was employed in three (numbers of order 7, 8 and 9) and produced satisfactory results in two, mainly because it allowed almost total equivalence of pronation-supination as far as the opposite side was concerned. Actually, wrist total arthrodesis, carried out by conventional techniques as the A.O. technique, does not interfere with the radioulnar articulation, consequently it does not correct occasional pronation-supination blocks. The conclusion is that the combination of these two techniques may be useful in these cases and must be indicated.

\section{CONCLUSION}

Although this sample is relatively small and contains different etiologies dearrangement cases of the distal raioulnar articulation, the results, though preliminary, are encouraging in relation to applicatibility and efficacy of the Sauvé-Kapandji technique to treat such difficult problems. This operation confirmedly is a valuable option mainly to treat young patients with trauma sequelae, when more radical procedures as Darrach's would be disabling. Not comparing it with other procedures as Bowers's for instance, the Sauvé-Kapandji operation is an effective therapeutic weapon, and it must be recommended for cases similar to those mentioned in this study. However, though relatively simple and easy to execute, it must be acurately carried out, in order to repeat our good results. 